\title{
Reproducibility of a hemi mandible positioning device and a method for measuring alveolar bone loss area in mice
}

\author{
Elken G. Rivaldo ${ }^{1,2)}$, Dalva M. P. Padilha ${ }^{1,3)}$, \\ Fernando N. Hugo ${ }^{4)}$, Juliana B. Hilgert ${ }^{5)}$ and Bruno R. Rybu ${ }^{6)}$ \\ ${ }^{1)}$ Geriatric and Gerontology Institute, Pontifical Catholic University, Porto Alegre, Brazil \\ ${ }^{2)}$ Faculty of Dentistry, Lutheran University of Brazil, Canoas, Brazil \\ ${ }^{3)}$ Faculty of Dentistry, Federal University of Rio Grande do Sul, Porto Alegre, Brazil \\ ${ }^{4)}$ Faculty of Dentistry of Piracicaba, State University of Campinas, Piracicaba, Brazil \\ ${ }^{5)}$ Graduate Program in Epidemiology, Federal University of Rio Grande do Sul, Porto Alegre, Brazil \\ ${ }^{6)}$ Faculty of Dentistry, Federal University of Rio Grande do Sul, Porto Alegre, Brazil
}

(Received 8 July and accepted 9 December 2006)

\begin{abstract}
The objective of this study was to determine the reproducibility of a positioning device and a method for measuring the area of alveolar bone loss (ABL) in hemi mandibles of mice. Mucoperiosteal flaps were raised in $\mathrm{CF}_{1}$ Mus domesticus mice $(\mathbf{n}=10)$ on the buccal aspects of the left side of the mandibles. Twenty-one days after surgery, the animals were sacrificed, and the mandibles were resected and stained with $1 \%$ toluidine blue. Two positioning devices, one for the buccal and the other for lingual aspect, were fabricated to keep the hemi mandibles in a standard position. The reliabilities of the positioning device and the method for measuring ABL area were analyzed using two series of pictures. The photographs were digitized and ABL measured as the exposed root area. Measurements were performed by two blinded examiners using image analysis software. Intra- and inter-examiner reproducibility of the positioning method ranged from -1.5 to $+1.33 \mathrm{~mm}$, while intra- and inter-examiner reproducibility of the measurement technique ranged from $\mathbf{- 3 . 3 7}$ to 14.70 . Our results indicate that, due to the small variation observed in ABL area assessment, the positioning device and method for measuring ABL area represent useful techniques for evaluating ABL in mice. (J. Oral Sci. 49, 13-17, 2007)
\end{abstract}

Correspondence to Dr. Elken Gomes Rivaldo, rua João Mora, 182, Porto Alegre, Rio Grande do Sul, CEP 91920-290, Brazil Tel: +55-51-32413337

Fax: +55-51-32413337

E-mail: elken@via-rs.net
Keywords: alveolar bone loss; result reproducibility; mice.

\section{Introduction}

Rodents are frequently used as experimental models for evaluation of alveolar bone loss. Several techniques based either on quantification or on scoring, have been proposed for measuring the alveolar bone loss in rodents (1-8).

Earlier studies, reporting quantification of alveolar bone loss, described techniques based on vertical measurements from the cementoenamel junction (CEJ) to the alveolar bone at points aligned with the cuspal vertexes. The samples were directly measured, with the aid of a ruler with millimeter markings (4), or on photographs (2), where the ABL was charted according to pre-defined scores. In other techniques, other reference points such as: the center of molar mesial and distal roots $(3,5,6)$, center of mesial roots only (7), or furcation areas (1) were used to measure the distance between the CEJ to the alveolar bone. The measurement of exposed root area was first described by GaegaufZollinger et al. (1) and was later improved $(8,9)$. Aiming to make both visualization of the exposed root area and the staining easier, substances such as Indian ink $(2,4), 1 \%$ methylene blue $(3,8), 1 \%$ toluidine blue (9) and silver nitrate $(1,3)$ were used.

Standardization of the positioning of hemi mandibles to be measured is essential, although several studies of alveolar bone loss in rodents were performed without any description of procedures to standardize positioning of 
samples or reproducibility of data analysis $(1,2,4-7)$. Furthermore, even slight changes in the position of a sample can cause significant distortions of the area of alveolar bone loss to be measured, compromising the reproducibility of the results. Reproducibility assessment is considered an important step for developing any methodology and usually includes intra-examiner and inter-examiner reproducibility analysis (10).

There is no evidence in the literature to show that a device for positioning hemi mandibles of mice and a methodology for measuring $\mathrm{ABL}$ area in the buccal and lingual aspects of these samples would produce reproducible results. Hence, the objective of this study was to determine the reproducibility of a positioning device and a methodology for measuring $\mathrm{ABL}$ area in hemi mandibles of mice.

\section{Materials and Methods}

\section{Study design and experimental procedure}

This experiment was carried out after the approval, and in accordance with the regulations, of the Ethics Committee of the Pontifical Catholic University of Rio Grande do Sul. Ten male $\mathrm{CF}_{1}$ Mus domesticus mice were used in this experiment. After weaning, the mice were kept in individual cages and they received distilled water and standard pellets of diet ad libitum during the three months and three weeks of the study. Standard conditions of light (12h light/dark cycle) and temperature (approximately $20^{\circ} \mathrm{C}$ ) were maintained during the experiment. Animals were monitored daily.

Three months after weaning, the mice were weighed and intramuscularly anesthetized [ketamine 100g/l (Dopalen, Agribrands do Brasil Ltda, Paulínia, Brazil) $+2 \%$ aqueous solution of 2-(2,6-xilidine)-5,6-dihydro-4- $H$-1,3-thiazine hydrochloride (Rompun, Bayer S.A., São Paulo, Brazil), in the ratio of $1: 1$, at a dosage of $1.0 \mathrm{ml} / \mathrm{kg}$ ]. A mucoperiosteal flap was raised on the buccal aspect of lower left molars. The mucosa was separated from the underlying bone after an incision at the marginal gingiva with the aid of a small elevator, and was immediately readapted, without any suture (11). The procedure was performed in approximately $40 \mathrm{sec}$. The right hemi mandible served as control. Animals received only water during the initial $24 \mathrm{~h}$ to avoid displacement of the flap. Twenty-one days after the surgical procedure, animals were sacrificed by cervical dislocation under anesthesia $(12,13)$.

\section{Sample processing}

Under a stereomicroscope, mandibles were divided along the medial line, cleaned of all soft tissues, and all organic material was removed with the help of $9 \%$ sodium hypochlorite applied with a brush. Specimens were then fixed in $10 \%$ buffered formalin for $12 \mathrm{~h}(12,13)$

Each hemi mandible was stained with $1 \%$ toluidine blue to disclose the exposed root area and the limits of enamel, cementum, and bone. Starting from the described procedure, two series of photographs were taken. In the first sequence (S1), designed to evaluate the performance of the positioning devices, one right hemi mandible and one left hemi mandible of the same animal were photographed 30 times each on the buccal and lingual aspects, adding up to 120 photographs. Every set of 30 pictures was completed after the right or left hemi mandible was inserted in the positioning device, photographed, removed from, re-inserted in the device and photographed again under the stereomicroscope for 30 times. In the second sequence (S2), designed to evaluate the reproducibility of the method for measuring $\mathrm{ABL}$ area, 10 right hemi mandibles had their buccal and lingual aspects photographed. All photographs were taken under magnification $(\times 2.5)$, having light and position standardized in a stereomicroscope (B201, Olympus, Tokyo, Japan) with conventional film (Kodak Ultra 135, Kodak Brasileira Comércio e Indústria Ltda, São Jose dos Campos, Brazil).

The buccal and lingual positioning devices were fabricated to keep buccal and lingual cusps aligned between themselves and in a perpendicular position to the long axis of the stereomicroscope's focus. A flat positioning device was made for photographing samples in the buccal aspect, whereas a modification was done to compensate for mandibular anatomy on the lingual aspect. In detail, this special positioning device consisted of a polyvinylsiloxane block and two $2 \mathrm{~cm}$-long metallic rods that were placed one $\mathrm{cm}$ apart from each other in the block in order to keep the samples in a standard position with the lingual and buccal cusps at the same level (Fig. 1). Fig. 2 shows an illustration of the positioning device showing an anterior angulation of $12^{\circ}$ and a lateral angulation of $5^{\circ}$.

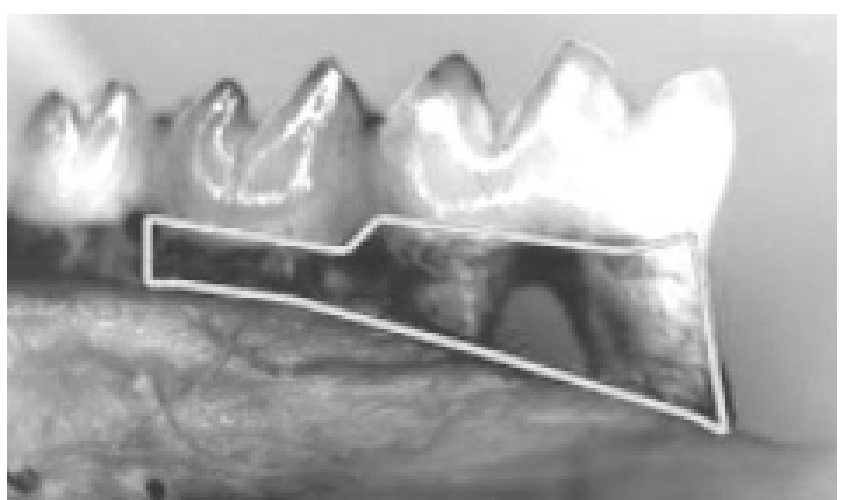

Fig. 1 The lingual aspect of mandible on the positioning device. Note that buccal and lingual cusps are in the same level. 


\section{Measurements}

Later, the photographs were digitized with a scanner and analyzed with image analysis software (UTHSCSA Image Tool version 2.02, UTHSCSA, San Antonio, USA). Two blinded examiners analyzed the digitalized pictures of the samples. Analyses were performed twice by each examiner with a two-week interval between the assessments of the areas of alveolar bone loss. The first examiner was an experienced researcher, while the second examiner was an undergraduate dental student with no previous experience in the assessment of alveolar bone loss, but who had been trained by the first examiner.

The alveolar bone loss area in the lingual aspect was measured at the first and second molars according to the reference points proposed by Tatakis and Guglielmoni (8) and modified by Rivaldo et al. (13) (Fig. 3A): mesially, by the mesial edge (CEJ to alveolar bone) of the mesial root of the first molar; coronally, by three points on each of the two molar teeth: two points defined by the position of the CEJ on the mesial and distal aspects of the tooth and one defined by the most apical position of the CEJ on the surface of the tooth; distally, by the distal edge (CEJ to alveolar bone) of the distal root of the second molar tooth; and apically, by the most apical position of the alveolar bone on the first and second molar mesial and distal root surfaces. The alveolar bone loss area in the buccal aspect was measured in the first molar according to the following reference points (Fig. 3B): mesially, by the mesial edge (CEJ to alveolar bone) of the mesial root of the first molar; coronally, by one point defined by the most apical position of the CEJ on the surface of the tooth; distally, by the distal edge (CEJ to alveolar bone) of the distal root of the first molar; and apically by the most apical position of the alveolar bone on the mesial and distal root surfaces. Results are shown in $\mathrm{mm}^{2}$.
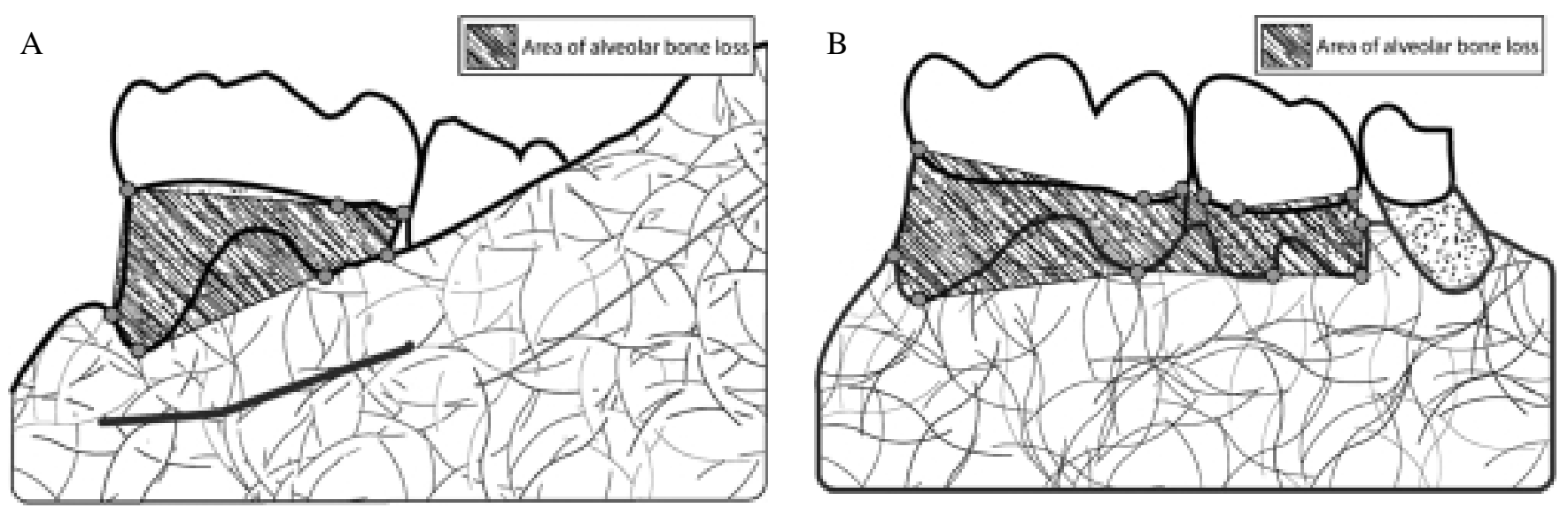

Fig. 3 Reference points for measuring alveolar bone loss area in the lingual (A) and buccal (B) aspects of the hemi mandibles.

\section{Statistical analysis}

The statistical approach used for reproducibility analysis of the alveolar bone loss area measurements used in our study was proposed by Bland and Altman (14). The analyses were performed with GraphPad Prism 4.0 (GraphPad Software, USA).

\section{Results}

Table 1 shows the reproducibility [and 95\% Confidence Intervals $(\mathrm{CI})]$ of the measurements, represented by the mean of the differences between pairs of measurements of ABL area, performed on the digitized images of both buccal and lingual aspects of the first series of photographs (S1). Intra- and inter- examiner reproducibility ranged, on an average, from -1.50 to 1.33 .

The reproducibility (and 95\% CI), represented by the mean of the differences between pairs of measurements of the alveolar bone loss on the second series of photographs

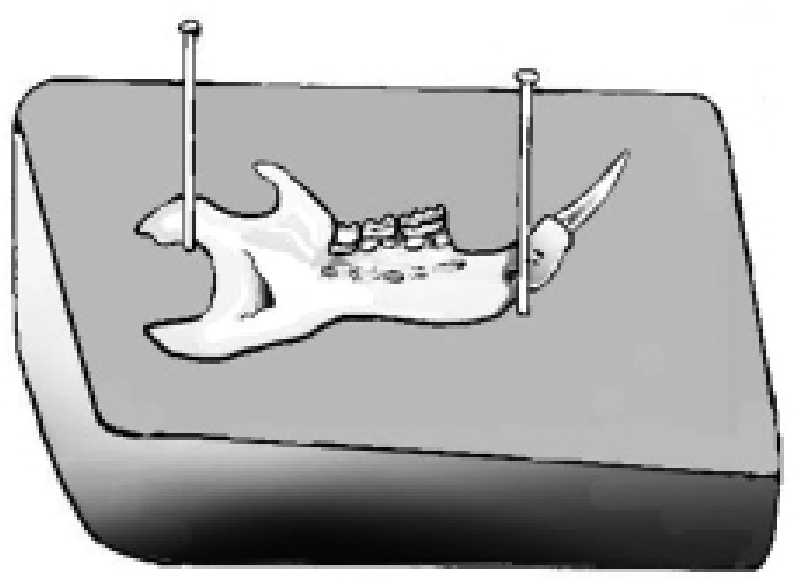

Fig. 2 Illustration of positioning device showing an anterior angulation of $12^{\circ}$ and a lateral angulation of $5^{\circ}$. 
(S2), are shown on Table 2. Intra- and inter- examiner reproducibility ranged, on an average, from -3.37 to 14.70 .

\section{Discussion}

The present study evaluated the reproducibility of a device for positioning hemi mandibles and of a method for quantification of alveolar bone loss (ABL) in mice. The results obtained in the $\mathrm{S} 1$ series of photographs regarding reproducibility of the device, without ABL variation in the sample, indicated that both the buccal and the lingual positioning devices allowed the placing of hemi mandibles in a position where the assessment of the ABL area could be performed with small variation (i.e. inter-examiner differences in measurements of ABL ranged from -1.03 to $+1.03 \mathrm{~mm}$ ). Similar results were also reported by Rivaldo et al. (13) and Tatakis and Guglielmoni (8), who reported the importance of placing the samples, mainly their lingual aspects, in a proper position (i.e. in a perpendicular position in relation to the microscope's focus) to avoid significant discrepancies in ABL area readings. Although standardization, in this case placing the samples properly to avoid inconsistencies in the measurements of ABL areas, is essential to avoid methodological flaws, it has not been reported by many researchers $(1,2,4-7)$.

The measurement of the ABL area only in first (buccal aspect) and in first and second molars (lingual aspect) is a modification of Tatakis and Guglielmoni (8) methodology for measuring $\mathrm{ABL}$ in rats. In our study, measurements were performed also in the buccal aspect of the samples because we observed, in a pilot study, that the variability of the readings was smaller when obtained from the buccal aspects of the samples. This was due to the fact that the areas analyzed were smaller when compared with ABL areas in the lingual aspects. Third molars were not analyzed due to their lingual inclination, as well as the frequent loss of these teeth observed in senescence accelerated mice (7). Furthermore, it is also important to ensure that the staining of the samples with $1 \%$ toluidine must be uniform, neither too dark nor too light, in order to make the limits of the CEJ and bone-cementum clearer and to simplify the process of analyzing the samples.

Although the measurements of ABL areas presented in this study were reproducible, we believe that capturing the images with a digital capturing system would be easier and produce images of better quality. By doing that, several steps such as using conventional photographic equipment and films, printing and scanning the photographs could be avoided.

Since there have been no other experiments that assessed the reproducibility of the positioning of hemi mandibles for ABL measures, no comparisons could be made. Our results showed that both S1 and S2 measurements were reproducible and within a small range (i.e. mean of the differences were usually small) (14). Furthermore, they confirm that the proposed positioning devices are effective for measuring the area of ABL in mice experiments.

Our findings confirm the results reported by Rivaldo et al. (13), based on Tatakis and Guglielmoni methodology

Table 1 Reproducibility (and 95\% CI) of alveolar bone loss area measurements in the S1 series of photographs, right and left hemi mandibles; buccal and lingual aspects

\begin{tabular}{|c|c|c|c|c|c|c|c|}
\hline & & \multicolumn{2}{|c|}{ Intra examiner 1} & \multicolumn{2}{|c|}{ Intra examiner 2} & \multicolumn{2}{|c|}{ Inter examiners } \\
\hline & & Buccal aspect & Lingual aspect & Buccal aspect & Lingual aspect & Buccal aspect & Lingual aspect \\
\hline \multirow{2}{*}{$\begin{array}{l}\text { Right mandible } \\
\text { (control) }\end{array}$} & $\begin{array}{c}\text { Mean of } \\
\text { differences }\end{array}$ & -0.38 & 0.53 & -0.02 & -1.50 & -1.03 & 1.03 \\
\hline & $95 \%$ CI & -2.24 to 1.47 & -1.21 to 2.27 & -1.41 to 1.37 & -4.28 to 1.27 & -4.77 to 2.72 & -1.19 to 3.25 \\
\hline \multirow{2}{*}{$\begin{array}{l}\text { Left mandible } \\
\text { (operated) }\end{array}$} & $\begin{array}{c}\text { Mean of } \\
\text { differences }\end{array}$ & 1.33 & 0.19 & -0.61 & -0.76 & -0.11 & 1.07 \\
\hline & $95 \% \mathrm{CI}$ & -2.86 to 5.51 & -2.27 to 2.65 & -3.77 to 2.56 & -3.61 to 2.09 & -4.14 to 3.93 & -2.12 to 4.27 \\
\hline
\end{tabular}

Table 2 Reproducibility of alveolar bone loss area measurements in the S2 series of photographs, right hemi mandible; buccal and lingual aspects $(\mathrm{n}=10)$

\begin{tabular}{ccccccc}
\hline & \multicolumn{2}{c}{ Intra examiner 1 } & Intra examiner 2 & \multicolumn{2}{c}{ Inter examiners } \\
\hline & Buccal aspect & Lingual aspect & Buccal aspect & Lingual aspect & Buccal aspect & Lingual aspect \\
$\begin{array}{c}\text { Mean of } \\
\text { differences }\end{array}$ & -1.03 & 8.89 & -2.84 & 14.70 & 3.45 & -3.37 \\
$95 \%$ CI & -9.01 to 10.09 & 53.94 to 64.05 & -20.46 to 14.79 & -51.01 to 80.41 & -9.20 to 16.19 & -51.61 to 44.88 \\
\hline
\end{tabular}


(8), indicating that the positioning devices allow correct visualization of the root exposed area, without distortion. Our results also indicate that the methodology for measuring $\mathrm{ABL}$ area and the positioning devices represent useful techniques for the study of $\mathrm{ABL}$ in mice, in both pathogenesis and therapeutic intervention experiments.

\section{References}

1. Gaegauf-Zollinger R, Burckhardt JJ, Guggenheim B (1982) Radiographic measurements of alveolar bone loss in the rat. Arch Oral Biol 27, 651-658

2. Gupta OP, Shaw JH (1956) Periodontal disease in the rice rat. II. Methods for the evaluation of the extent of periodontal disease. Oral Surg Oral Med Oral Pathol 9, 727-735

3. Klausen B, Evans RT, Sfintescu C (1989) Two complementary methods of assessing periodontal bone level in rats. Scand J Dent Res 97, 494-499

4. Keyes PH, Gold HS (1955) Periodontal lesions in the Syrian hamster. I. A method of evaluating alveolar bone resorption. Oral Surg Oral Med Oral Pathol 8, 492-499

5. Messer HH, Douglas WH (1980) Inhibition by chlorhexidine of alveolar bone loss in mice. $\mathrm{J}$ Periodontal Res 15, 646-649

6. Moriya Y, Ito K, Murai S (1998) Effects of experimental osteoporosis on alveolar bone loss in rats. J Oral Sci 40, 171-175
7. Sashima M, Satoh M, Suzuki A (1990) Alveolar bone loss of senescence-accelerated mouse (SAM). J Dent Res 69, 82-86

8. Tatakis DN, Guglielmoni P (2000) HLA-B27 transgenic rats are susceptible to accelerated alveolar bone loss. J Periodontol 71, 1395-1400

9. Hilgert JB, Hugo FN, Bozzetti MC, Padilha DMP (2003) Comparison of two techniques to measure alveolar bone loss in rodents. J Dent Res 82, Spec Iss C, 259 (abstract)

10. Hulley SB (2001) Designing clinical research: an epidemiological approach. 2nd ed, Lippincott \& Williams \& Wilkins, Philadelphia, 55-66

11. Binderman I, Adut M, Zohar R, Bahar H, Faibish D, Yaffe A (2001) Alveolar bone resorption following coronal versus apical approach in a mucoperiosteal flap surgery procedure in the rat mandible. J Periodontol 72, 1348-1353

12. Yaffe A, Fine N, Binderman I (1994) Regional accelerated phenomenon in the mandible following mucoperiosteal flap surgery. J Periodontol 65, 7983

13. Rivaldo EG, Padilha DP, Hugo FN (2005) Alveolar bone loss and aging: a model for the study in mice. J Periodontol 76, 1966-1971

14. Bland JM, Altman DG (1986) Statistical methods for assessing agreement between two methods of clinical measurement. Lancet 1, 307-310 